\title{
MOLECULAR STRUCTURE OF STRESS GRANULES AND THEIR ROLE IN THE EUKARYOTIC CELL
}

\author{
Paulina Pietras ${ }^{1}$, Marta Leśniczak ${ }^{1}$, Mateusz Sowiński ${ }^{1}$, Witold Szaflarski ${ }^{1}$
}

\begin{abstract}
Stress granules (SGs) are cytoplasmic structures found in eukaryotic cells, from yeast to human cells. They are made up of proteins, RNA and small ribosome subunits (40S). They arise as a result of the rapid shutdown of active protein biosynthesis in the cell, which is the result of the appearance of a stress factor. The mechanism of regulation of protein biosynthesis in response to stress takes place at two control nodes: (1) phosphorylation of the $\alpha$ subunit of the eIF2 factor as a result of the action of stress-recognizing kinases or by modulation of the mTOR pathway activity, which regulates the initiation of protein biosynthesis by the formation of a complex within the so-called cap structure. The protein arrest causes aggregation of the translation process components and other cell components (other proteins or mRNA molecules) into SGs. A lot of data indicates the active participation of SGs in metabolic processes, their control role over pro- and anti-apoptotic processes as well as in the development of cancer, neurodegenerative diseases and their defensive role in viral infections.
\end{abstract}

Running title: Stress granules in the cell

Keywords: stress granules, ribosome, rRNA, protein biosynthesis, anticancer drugs, cancer 


\section{Introduction}

Stress granules (SGs) are structures that contain proteins and RNA [1]. They appear in the cytoplasm of eukaryotic cells, both spontaneously and in response to stress, which may be chemical or physical (e.g. heat stress) [2]. The first reports describing SGs come from 1983. In cultures of Peruvian tomatoes, under the influence of heat stress, aggregates were formed in the cytoplasm that contained proteins with low molecular weight [3]. The development of immunofluorescence methods allowed for a more detailed analysis of SGs and their compounds in mammalian and cancer cells (Fig. 1), also subjected to heat stress [4]. The size of SGs varies depending on the type of stress and ranges from 0.1 to $1 \mu \mathrm{m}$. SGs are membrane-less structures that distinguish them from structures such as the nucleus or mitochondria and bring them closer to structures such as the nucleoli. SGs can be observed quite conveniently by immunofluorescence methods in the in vitro stressed cells. One of the first observations of SGs was made in the 1990s, and the pioneers in these studies were Nancy Kedersha and Paul Anderson from Harvard Medical School in Boston, USA. They showed that SGs are composed of mRNA and proteins such as PABP, TIA- 1 and TIAR, and are formed as a result of phosphorylation of the $\alpha$ subunit of eIF2 by kinases that detect various types of stress [5].

\section{Formation of stress granules}

SGs are formed as a result of a sudden inhibition of protein biosynthesis on the ribosomes in all types of eukaryotic cells, both normal cells that build tissues, organs and entire organisms, and pathologically changed (cancer and neurodegenerative diseases). A properly functioning cell requires, among others, structural and enzymatic proteins. Ribosomes, which catalyze the formation of a peptide bond between amino acids according to the sequence encoded in the mRNA, are responsible for
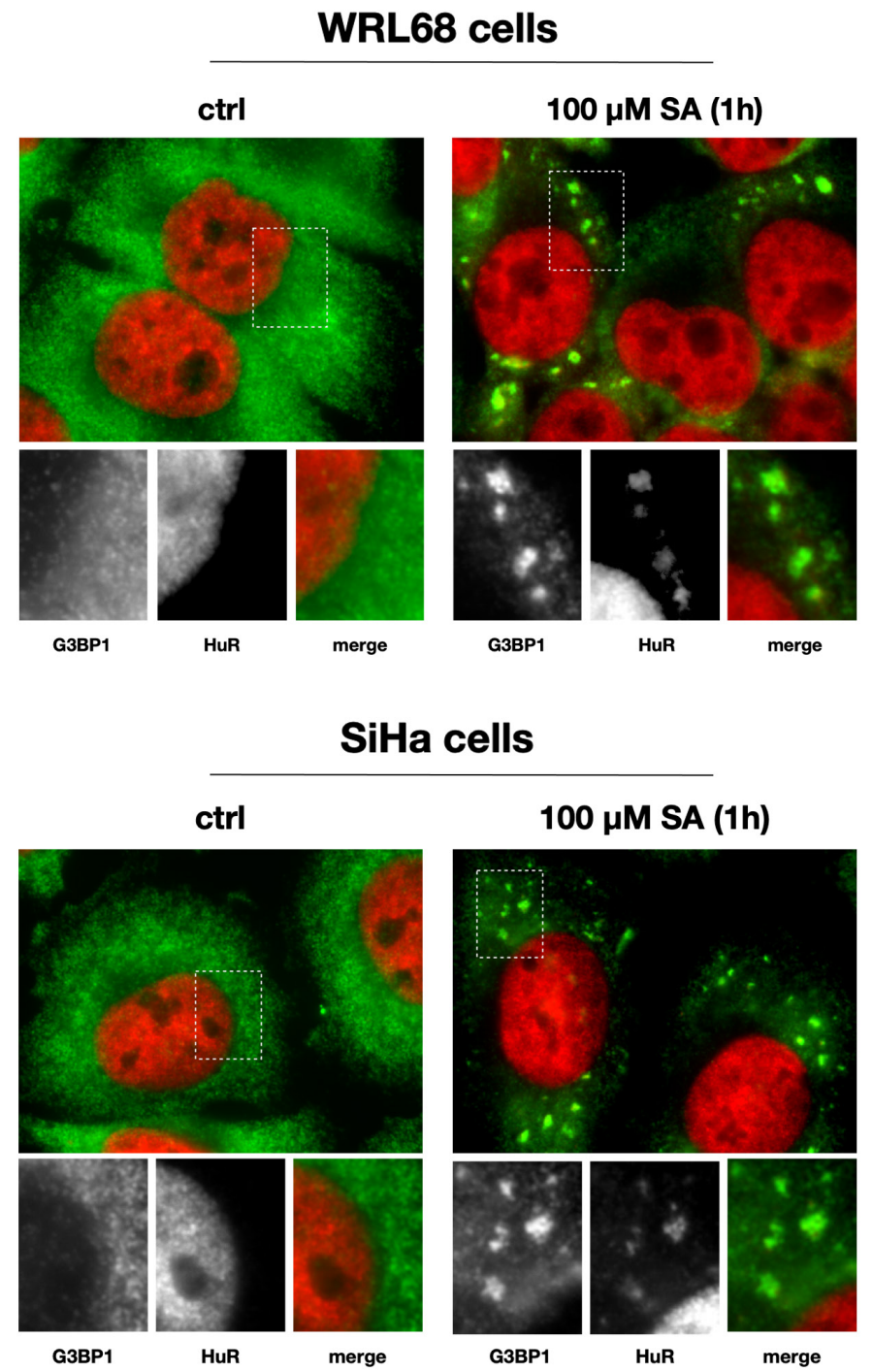

FIGURE 1 An example of stress granules formation in two types of cells (WRL68 and SiHa) using immunofluorescence method. This figure shows two stress granules markers: G3BP1 and HuR as well as nucleus in cells under normal growth conditions (ctrl) and the formation of stress granules under stressful conditions caused by sodium arsenite (SA) 
their formation. Protein biosynthesis is a process closely controlled by various molecular mechanisms in the cell.

SGs result from the rapid shutdown of protein biosynthesis. There are two main pathways of regulating protein synthesis, both related to the initiation of protein synthesis. One is due to the activity of kinases that phosphorylate the serine residue at position 51 in the $\alpha$ subunit of the protein biosynthesis initiator ( $\mathrm{IF} 2 \alpha$ ), while the other is associated with the formation of the eIF4F complex responsible for binding mRNA to the ribosome.

Phosphorylation of eIF $2 \alpha$ is recognized as the primary mechanism for the overall inhibition of cellular protein synthesis [5]. The initiating factor eIF2 is composed of three subunits, i.e. $\alpha, \beta$ and $\gamma$, and forms two functional complexes: active eIF2·GTP and inactive eIF2-GDP. The active complex binds the initiator tRNA carrying the amino acid methionine (Met-tRNAi) and together forms a ternary complex (TC), which binds to a small ribosome subunit (40S) and mRNA, in the area of the so-called the P (peptidyl) site. In the next step of protein biosynthesis initiation, the simultaneous binding of the large ribosome subunit and the hydrolysis of GTP and GDP takes place. The inactive eIF2-GDP complex is released. The eIF2B factor is responsible for the regeneration of the inactive eIF2-GDP complex.

The eIF2B factor is quantitatively about $20 \%$ of the eIF2 factor pool, which is sufficient for effective regeneration of the eIF2-GDP complex. Thus, during regeneration, the eIF2 -GDP · eIF2B complex is formed temporarily. Studies have shown that the phospho-eIF2 ( $\alpha$-phospho)·GDP-eIF2B complex is much more stable compared to the eIF2-GDP·eIF2B complex, which reduces the availability of eIF2B. The appearance of eIF2 ( $\alpha$-phospho) molecules in the same amount as eIF2B or more results in blocking the protein biosynthesis at the initiation stage.

\section{How is eIF $2 \alpha$ phosphorylation regulated?}

Four kinases are present in mammalian cells that are capable of phosphorylating a key serine at position 51 on the $\alpha$-subunit of eIF2. All four kinases, i.e. HRI, GCN2, PKR and PERK, are responsible for monitoring homeostasis in the cell and detecting stress stimuli [6].

The HRI kinase is responsible for detecting oxidative (oxygen), osmotic and thermal stresses as well as for limiting haem access. Historically, it was the detection of heme restriction in erythrocytes that became the basis for naming this kinase (Heme-regulated eIF $2 \alpha$ kinase). In the case of heme deficiency in reticulocytes, the inhibition of protein synthesis correlates with the activation of HRI [7]. The conducted studies have shown that sodium arsenite $\left(\mathrm{NaAsO}_{2}\right)$, a commonly used stress granule activator, stops protein biosynthesis by phosphorylating serine 51 in eIF $2 \alpha$ via HRI kinase [8].
The GCN2 kinase is an amino acid level sensor. It binds to amino acid-free tRNA molecules. Therefore, it participates in the regulation of metabolism in response to the lack of nutrients. The GCN2 kinase itself is a multidomain protein, and each of these domains may be responsible for a different function of the protein. The C-terminus domain of the GCN2 protein has high homology to histidyl RNA synthetase (the enzyme responsible for bonding between the tRNA encoding histidine and amino acid histidine). Thus, binding of GCN2 to tRNA lacking an amino acid causes a conformational change in GCN2 itself (domains inside this protein change the angle with each other by 180 degrees), which activates this protein kinase and consequently, it phosphorylates serine 51 in the factor eIF $2 \alpha$ [9]. Thus, the deficiency of amino acids stops protein biosynthesis, which is an important regulator of homeostasis, since protein biosynthesis is a high energy-consuming process in the cell. Intensively dividing bacterial cells allocate approx. $80 \%$ of their resources to protein biosynthesis, and eukaryotes - approx. 20\%.

The PKR kinase is activated in response to the emergence of double-stranded RNA molecules (dsRNA). It is mainly one of the mechanisms of protection against viruses, the genetic material of which may be RNA. The PKR protein contains a dsRNA binding domain at its $\mathrm{N}$ (amino) terminus, abbreviated as dsRBD. The dsRBD domain consists of two tandem copies of a conserved double-stranded RNA-binding motif, i.e. dsRBM1 and dsRBM2. The second domain in the PKR protein is a kinase. As with the GCN2 protein, here also the binding of dsRNA by the dsRBD domain of the double-stranded RNA causes conformational changes in the kinase domain which becomes active and can phosphorylate serine 51 in the factor eIF $2 \alpha$. The consequence of stopping protein biosynthesis here is preventing the translation of viral proteins.

In addition to the phosphorylation of serine 51 of eIF $2 \alpha$, another method of translation control is the activation of the eIF4F complex, the formation of which depends on the mTOR signaling pathway. The formation of the eIF4F complex is preceded by the attachment of the mRNA to the ribosome. The complex itself consists of mRNA and proteins that initiate protein biosynthesis, i.e. eIF4E, eIF4A and eIF4G. The eIF4G factor is the scaffold for the whole complex, eIF4A is the helicase that binds mRNA. eIF4E is the factor that binds to the mRNA cap structure that is characteristic of most genes. Under normal conditions, eIF4E binds to the mRNA cap structure and forms, together with other factors, the complete eIF4F complex. However, eIF4E also interacts with 4E-BP proteins, which are phosphorylated by mTORC1 kinase, a major player in the mTOR signaling pathway. 4E-BP proteins, which are phosphorylated, are not active and cannot bind eIF4E. Thus, this state of affairs normally occurs 
when the cell has access to nutrients and the mTOR pathway is active. In the case of energy deficiencies, the mTOR pathway is turned off and the 4E-BP1 proteins are dephosphorylated, which ultimately leads to the binding of eIF4E and the breakdown of the entire eIF4F complex. The breakdown of the eIF4F complex blocks the initiation of protein biosynthesis and prevents the expression of certain cap-dependent genes.

Research has shown that SGs can arise as a result of stopping translation initiation through both of the above mechanisms (Fig. 2). One of the most widely used SGs activators is sodium (III) arsenite, which activates HRI kinase, which phosphorylates serine 51 in eIF $2 \alpha$. It is a classic mechanism of SGs activation by eIF $2 \alpha$ phosphorylation. It turned out that hydrogen peroxide $\left(\mathrm{H}_{2} \mathrm{O}_{2}\right)$ causes the formation of SGs by stopping protein biosynthesis by breaking down the eIF4F complex [10]. Additionally, such granules did not have all the proteins present in those activated with sodium arsenite, i.e. PABP, eIF4E, eIF4G and eIF3b. Studies on the anti-cancer drug vinorelbine have shown that induction of SGs formation by this drug requires both eIF $2 \alpha$ phosphorylation (PERK activation) and 4E-BP1 protein dephosphorylation (mTOR exclusions) [11]. Today we know many substances that cause the formation of SGs. The most common ones in the literature include sodium arsenite (activation of HRI kinase), thapsigargin [12], vinorelbine (PERK kinase activation) [11], hydrogen peroxide [10] and nitric oxide [13]. The latter also creates slightly different SGs in which the eIF3b factor is absent and which are pro-apoptotic [13].

\section{Structural composition of stress granules}

It is well established that SGs consist of RNA and proteins [14]. Database covering stress granule protein composition has recently become available at https://msgp.pt. According to its statistics, a total of 464 proteins are reported to be recruited to SGs [15]. The most significantly enriched group represented by 252 proteins has been classified as "RNA-binding". The remaining proteins have been grouped

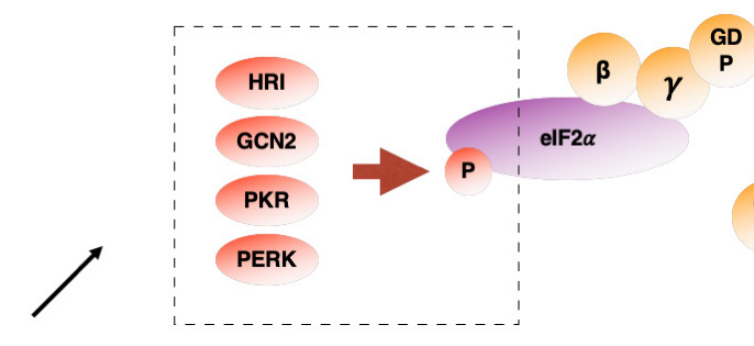

stress

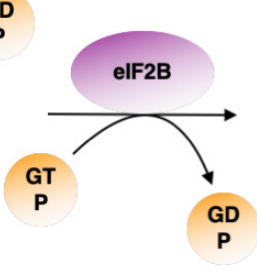

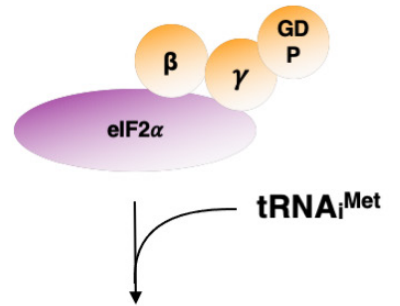

Ternary complex
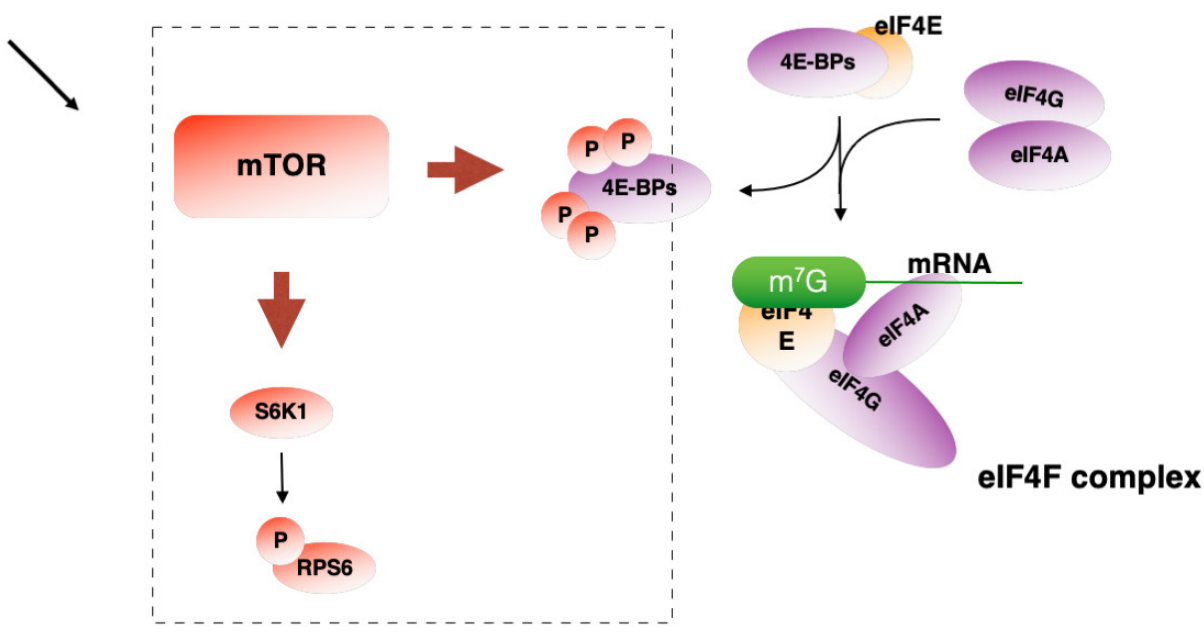

FIGURE 2 Two mechanisms for regulating protein biosynthesis. The top diagram shows the mechanism based on the phosphorylation of the eIF $2 \alpha$ factor, which is involved in the correct binding of the initiator tRNA to the ribosome. When one of the kinases is activated, the eIF $2 \alpha$ factor is phosphorylated, which leads to blocking the formation of the triple complex and stopping the initiation process. The second mechanism is related to the mTOR signaling pathway, which regulates the phosphorylation of 4E-BP and RPS6 (ribosomal protein S6) proteins. Under normal growth conditions, mTOR phosphorylates 4E-BP proteins, preventing them from binding to eIF4E, whereby the latter may form the active eIF4F initiation complex. Inhibition of mTOR activity causes hypophosphorylation of the 4E-BP proteins which bind to eIF4E in this state, thereby blocking the formation of the eIF4F complex 
into several other categories, such as "proteins with antioxidant activity", "signal transducers", "translation regulators", and "transport proteins". However, in SG's- related research field, immunofluorescence microscopy remains a gold standard. It allows for the identification of proteins considered as Stress Granule markers. These are G3BP1, TIA-1, TIAR and FxR1. Historically, TIA-1 and TIAR were described first [5]. They are located in the cell nucleus but can move to the cytoplasm and end up in SGs upon stress exposition.

G3BP1 protein is a well-established SG marker-protein [16]. It has been proved that the expression of G3BP1 arrests SG formation whereas overexpression of the G3BP1 protein itself leads to the spontaneous formation of SGs in the absence of stress[17, 18]. However, recent data shows that only the G3BP1/G3BP2 double knockout prevents the formation of SGs [18].

Well-known RNA-binding markers widely used in the SG assembly research field are PABP and HuR [17]. PABP binds directly to the poly(A) tail in mRNA whereas HuR selectively binds AU-rich motifs. While the principal function of the HuR protein remains unclear it has recently been described as an adipogenesis inhibitor [19]. Mechanistically, HuR can inhibit adipogenesis by recognizing and modulating the stability of hundreds of adipocyte transcripts, including Insig1, a negative regulator of adipogenesis. The general purpose of SGs assembly is protein sequestration, which limits proteome availability and its function in the cytoplasm. Sequestration of the HuR protein into SGs could therefore act as a scavenger of its cytoplasmatic activity.

FXR1 is an SG marker with a molecular weight of $69 \mathrm{kDa}$. It is a homolog of the FMRP protein, which in the presynaptic part acts as a translation repressor regulating local protein synthesis in nerve cell [20]. Closely related to FXR1 is Caprin-1 protein. Phase separation studies in the context of translation regulation and post-translational modification in neurodegenerative diseases have proven that there is an interaction between FMRP and the Caprin-1 protein, which regulates the translation of at least two known mRNA molecules, including the CaMKII $\alpha$ gene and Map1b $[21,22]$.

\section{Participation of stress granules in the cell signaling}

Once assembled SGs interact with proteins involved in many signaling pathways, influencing cellular metabolism globally [23]. SGs consist of structural proteins and enzymes such as ubiquitin-modifying enzymes, protein kinases, phosphatases, helicases, glucosyltransferases, methyltransferases, and GTPases. These can be further grouped into 2 categories: proteins that affect the formation of SGs and proteins that inhibit signal transduction pathways without affecting the process of granule formation, such as TRAF2 and RACK1.
Viral infection-induced phosphorylation of eIF $2 \alpha$ is the main switch initiating SG formation in mammalian cells [24]. Proteins such as Carpin 1, USP10, G3BP, HRI, YB-1, and eIF $2 \alpha$ are reported to be directly and indirectly affected by interaction with OGFOD1 [25]. Both granule formation and eIF2 $\alpha$ phosphorylation levels are elevated in cells with increased mRNA levels of this protein. OGFOD1 is therefore suspected to promote HRI dependent eIF $2 \alpha$ phosphorylation.

Several processes such as apoptosis, cell motility, and adhesion are strongly affected by a protein called RACK1 [26]. It participates in eIF3 complex binding and plays a crucial role in $40 \mathrm{~S}$ small ribosome subunit formation. It also triggers apoptosis through p38/JNK pathway activation and serves as a scaffold protein for MTK1 which acts upstream of this pathway. This process is inhibited upon stress when RACK1 is being bound by SG [27].

mTOR kinase consists of two distinct complexes (TORC1 and TORC2) that control cell growth and metabolism [28]. In human and yeast cell lines, SGs formation alters TORC1 signaling by sequestering both TORC1 and downstream kinases resulting in altered signaling during stress. Upon lack of amino acid supply stress, inactivated TORC1 accumulates in SGs in response to elevated eIF $2 \alpha$ phosphorylation levels. This process also involves the DYRK3 protein, which delays the disintegration of SGs and the related slowing down of the release of the active TORC1 kinase. Interestingly, DYRK3 may also promote mTOR activation through phosphorylation and deactivation of the TORC1 inhibitory subunit - PRAS40 causing SGs assembly inhibition. Thus, DYRK3 regulates TORC1 activation by slowing down SGs disassembly and through PRAS40 phosphorylation [29]. TORC1 activation is also dependent on the PABP- Pbp1 interaction [30]. Both Pbp1 overexpression and heat shock induce the sequestration of TOR and KOG1 / RAPTOR into SGs. This correlates with the inactivation of TORC1, regulated by phosphorylation of the Sch9/RPS6 downstream kinase.

Processes associated with microtubule dynamics, endosomal vesicle mobility and progression of the cell cycle are severely affected by Rho GTPases activity [31]. Upon activation, RhoA binds and activates ROCK1, which phosphorylates JNK-interacting protein 3 (JIP-3), causing JNK activation and induction of apoptosis. Thus, the sequestration of RACK1 in SGs prevents JNK-induced apoptosis [32].

Rho GTPases are also effectors of the Wnt signaling pathway, which inhibits SGs formation by binding directly to G3BP1 [33].

\section{Participation of stress granules in cancer diseases}

SGs are often found in areas of a tumor exposed to stress, in this case, the stress is most often associated with administered anticancer drugs [34, 35]. 
The formation of SGs may also result from the dysregulation of certain signaling pathways in neoplastic diseases [36]. Although the role of SGs in cancer is poorly understood and may vary depending on the type of cancer, different studies suggest that the formation of SGs is usually associated with different stages of tumor development, metastasis, and the acquisition of resistance by cancer cells under anti-cancer drugs treatment [37, 38]. Additionally, the fact that the components of SGs are involved in such cellular processes as angiogenesis, inhibition of apoptosis, regulation of the cell cycle and many other may prove the role of SGs in the development of the neoplastic disease itself $[26,39,40]$. Cancer cells, due to high metabolic activity and high proliferative potential, are usually exposed to many stress factors, such as hypoxia, nutrient deficiency or stress of the endoplasmic reticulum [41]. All of these factors can result in the formation of SGs, which in turn may facilitate the adaptation of neoplastic cells to stressful conditions. The formation of SGs promotes cell survival, in part by sequestering pro-apoptotic molecules, which leads to inactivation of the pathways leading to apoptosis [26]. SGs have been shown to be activated by mutant KRAS pancreatic cancer cells under stress to enhance tumor cell survival [42].

Adjibade et al. showed that sorafenib, which is a tyrosine kinase inhibitor and is used in the treatment of hepatocellular carcinoma, strongly induces SGs formation which has been associated with inhibition of translation initiation. In the mechanism of the response to sorafenib, PERK has been shown to be responsible for the phosphorylation of eIF $2 \alpha$ in the formation of SGs [43].

Treatment of C6 and U87MG malignant glioma cells with bortezomib, cisplatin and etoposide resulted in the formation of SGs in a manner dependent on eIF $2 \alpha$ phosphorylation at position 51 . In contrast, blocking the eIF $2 \alpha$ pathway resulted in increased chemotherapeutic induced cell death [44]. In turn, studies by Bittencourt et al. confirmed that the G3BP1 knockdown reduces the formation of SGs and stimulates bortezomib-induced apoptosis in U87 cells in vitro, as well as inhibits glioblastoma-induced angiogenesis in vivo. [37].

In many types of cancer, different components of SGs are expressed at a higher level than in normal tissue. In sarcomas, increased G3BP1 expression correlates with lower survival, while inhibition of the G3BP1 expression in sarcoma xenografts prevented SGs formation in vivo and tumor invasion, and completely blocks lung metastasis in mouse models. Additionally, Somasekharan et al. demonstrated that the YB-1 protein binds to and translationally activates the 5' untranslated region (UTR) of the G3BP1 mRNA, which promotes the formation of SGs [38]. Also in gastric cancer, G3BP1 is elevated and correlates with a poorer response of pa- tients to adjuvant chemotherapy. In addition, it was shown that the knockdown of this protein caused an increase in the expression level of other proteins involved in the pro-apoptotic pathways and sensitization of cells to the therapeutics used. Zhao et al. also indicated the YWHAZ protein, the high level of expression of which, together with the increased expression of G3BP1, results in worse response to chemotherapy among patients [45].

Adjibade et al. found that lapatinib, which is used in the treatment of HER2-positive breast cancer, causes the formation of SGs as a result of the phosphorylation of eIF $2 \alpha$ by PERK kinase. On the other hand, cells with silenced PERK expression are more sensitive to the chemotherapeutic agents used [40].

There is also growing evidence that the activity of several key components of SGs is dysregulated in pre-neoplastic states and intestinal tumors, which may indicate the potential role of SGs in the occurrence of colorectal cancer (discussed in [46]).

\section{Participation of stress granules in neurodegenerative diseases}

Recent studies have provided evidence that SGs can play an important role in neurodegenerative diseases. It has been shown that proteins associated with this type of diseases, some of which are RNA-binding proteins, are recruited to SGs or regulate their formation [47]. In addition, it has also been shown that the marker proteins of SGs are part of pathological inclusions [48].

One of the SGs proteins associated neurodegeneration is TDP-43 (DNA binding TAR protein 43), which is a highly conserved RNA binding protein. This protein plays an important role in alternative splicing and transcription regulation, and is responsible for the stabilization and transport of mRNA in the cytoplasm. $[49,50]$. It is recruited into SGs as a result of the action of various stressors, such as osmotic stress, oxidative stress, heat shock or viral infection, and also influences their dynamics [51, 52]. In vitro studies carried out on cell lines with silent expression of TDP-43 showed that the initial SGs formation, subsequent aggregation into larger structures, and ultimately the disintegration of SGs after stress relief were impaired compared to wildtype cells [53]. TDP-43 was found to form ubiquitinated cytoplasmic inclusions in the neurons of patients suffering from amyotrophic lateral sclerosis (ALS), fronto temporal degeneration, and in inclusion myositis $[54,55]$. Khalfallah et al. based on in vitro studies on astrocytes and cortical neurons, hypothesized that aging may affect the dynamics of SGs, which may result in the development of age-related neurodegenerative diseases [56].

FUS mutations associated with ALS, especially those associated with the nuclear localization signal (NLS), cause it to sequestrate in SGs after exposure to a stress stimulus [57]. In turn, in models show- 
ing the overexpression of mutant FUS, recruitment to SGs was demonstrated regardless of the presence of the stressor [58,59]. Additionally, Dormann et al. demonstrated the presence of SGs markers, such as PABP-1 or eIF4G in cytoplasmic FUS inclusions, which may indicate the role of SGs in the formation of FUS aggregates in patients with ALS [60]. Other SGs markers have also been identified as components of pathological aggregates that contribute to neurodegeneration, incl. TIA-1, HuR, eIF4G, ataxin-2, eIF3 in TDP-43 / FUS inclusions in amyotrophic lateral sclerosis and frontotemporal degeneration; TIA- 1 in Tau and Htt (polyQ) deposits in Alzheimer's and Huntington's diseases; ataxin-2 in TDP-43 inclusions in spinocerebellar ataxia (discussed in: [48]).

These studies provide evidence for the involvement of SGs in the development of neurodegenerative diseases. The initial sequestration of aggregation prone proteins followed by their excessive accumulation may cause amyloidogenic interactions to lead to the formation of persistent pathological oligomers and fibrils and subsequent disease development.

\section{Effect of stress granules on the viral infection}

Viruses are a kind of cellular stress. They have also been shown to activate the formation of SGs in cells. The first evidence of an interaction between viruses and SGs was found in 2002 [61]. Following viral infection, the cell produces a strong antiviral response to create an unfavorable environment for virus replication. Part of the innate immune response to infection is activation of the PKR stress kinase, which phosphorylates the eIF2 $\alpha$ subunit. Blocked viral replication is caused by translation inhibition, and consequently SGs are also formed. There are growing reports suggesting that Integrated Stress Response (ISR), and SGs in particular, contribute to antiviral defense [62].

It appears that the condensation state, composition and function of SGs may change over time as viral infections progress. Several RNA viruses have been shown to actively disrupt SGs build-up by breaking down their components. An example is the poliovirus (PV), which on the one hand activates the formation of SGs in the early stages of infection, but on the other hand also activates their breakdown in the further stages of infection by cleavage the G3BP1 protein in the C-terminal region by viruses [63]. Very similar results were obtained for the Encephalomyocarditis Virus (EMCV) [64].

In contrast to RNA viruses, the regulation of SGs formation during infection with DNA viruses is poorly understood. It appears that human cytomegalovirus (HCMV) infection modifies the unfolded protein response (UPR) and activates PERK kinase limiting the amount of phosphorylated eIF $2 \alpha$ to maintain translation [65].
The multiple mechanisms by which viruses inhibit or induce SGs may pose problems in the development of antiviral drugs that target SGs [62].

\section{Stress granules and anticancer therapy}

In recent years, there has been a global improvement in the survival rate of patients with diagnosed cancer. However, these diseases are still a big challenge in modern medicine, because one of the biggest problems is the resistance of cancer cells to administered drugs and the subsequent metastasis. So far, the search for changes at the molecular level has been effective, contributing to a better classification of tumors and the development of effective targeted therapies [66]. Unfortunately, not all patients will respond to a particular treatment strategy or become resistant to a given therapy, which means that there is a great need to find a treatment that can counter the drug-induced resistance mechanisms.

As described earlier, SGs are versatile and important regulators of many physiological functions, including cancer development, and there has been considerable interest in the role of SGs in treating cancer. SGs promote the survival of cells under stressful conditions, minimize energy expenditure, and at the same time regulate homeostasis in the area of RNA and proteins [67]. Research has shown that the formation of SGs is strongly related to the proliferation and metastasis of cancer cells. In addition, it turns out that some drugs used in chemotherapy, including 5-fluorouracil, bortezomib, or alkaloids such as vinorelbine, vincristine or vinblastine cause the formation of SGs $[11,68]$. On the one hand, in cancer treatment, the formation of SGs can lead to cell survival and increase cell resistance to chemotherapeutic drugs [69]. The combined use of drugs that inhibit the formation of SGs or promote their breakdown with chemotherapeutic drugs can reduce drug resistance. On the other hand, some drugs can enhance the effect of chemotherapy by inducing SGs controlled apoptosis. Moreover, the persistence of the granule leads to irreversible pathogenesis, especially in neurodegeneration and the aging process. Interfering with the specific recruitment of granule components or microtubule polymerization, as well as other mechanisms that may inhibit SGs formation, may inhibit tumor progression. Targeting SGs by disrupting their formation or the movement of their components may not only affect tumor progression, but also sensitize cancer cells to chemotherapeutic agents, may prove to be a new strategy in the treatment of cancer, neurodegenerative diseases and other chronic diseases [70]. SGs seem to be important factors of cancer cells in responce to various stresors and may be potential targets in preventing of cancer, progression and also chemoresistance development. Hence, further research is needed to understand role of SG in 
the development of aggressive and invasive types of cancer and how to treat them. This can help especially in patients who are refractory to certain therapeutic strategies.

\section{Conclusions}

SGs have been systematically studied for over 20 years. Every year, more and more data were obtained describing their role in normal and pathological conditions. Research has shown that SGs are involved in many physiological processes, and what is more, they can control them. The composition of SGs is very diverse, but the basic elements that build it are proteins and RNA. Within a protein group, various of these molecules can bind to the granule, which modifies the function of the protein as it becomes absent from the cytoplasm. The mechanisms of protein sequestration into SGs are unknown. The role of SGs themselves is not fully understood. The question of the presence of SGs in neoplastic cells is very interesting. We should ask here in two ways both about the participation of SGs in the process of neoplasm and its cancer, as well as about the participation of these structures in the resistance of cancer cells to chemotherapy. Asking the question like this opens a new way to analyze SGs and learn about their functions in cells. Fortunately, more and more advanced techniques of cellular and molecular biology allow for more and more perfect and detailed analysis of the nature of SGs in the cell.

\section{Ethical approval}

The conducted research is not related to either human or animal use.

\section{Acknowledgements}

This work was supported from National Science Center, Poland (grant no. 2018/30/E/NZ7/00614).

\section{Corresponding author}

Witold Szaflarski, Department of Histology and Embryology, Poznań University of Medical Sciences, 6 Święcickiego St., 60-781 Poznań, Poland, e-mail: witold@ump.edu.pl.

\section{Conflict of interest statement}

The authors declare they have no conflict of interest.

\section{References}

1. Kedersha N, Anderson P. Mammalian stress granules and processing bodies. Methods Enzymol. 2007;431:61-81; DOI: 10.1016/ S0076-6879(07)31005-7.

2. Mokas S, Mills JR, Garreau C, Fournier MJ, Robert F, Arya P, et al. Uncoupling stress granule assembly and translation initiation inhibition. Mol Biol Cell. 2009;20(11):2673-83; DOI: 10.1091/mbc.E08-10-1061.

3. Nover L, Scharf KD, Neumann D. Formation of cytoplasmic heat shock granules in tomato cell cultures and leaves. Mol Cell Biol. 1983;3(9):1648-55; DOI: 10.1128/mcb.3.9.1648

4. Arrigo AP, Suhan JP, Welch WJ. Dynamic changes in the structure and intracellular locale of the mammalian low-molecular-weight heat shock protein. Mol Cell Biol. 1988;8(12):5059-71; DOI: 10.1128/ mcb.8.12.5059.

5. Kedersha NL, Gupta M, Li W, Miller I, Anderson P. RNA-binding proteins TIA- 1 and TIAR link the phosphorylation of eIF- 2 alpha to the assembly of mammalian stress granules. J Cell Biol. 1999;147(7):1431-42;
6. Donnelly N, Gorman AM, Gupta S, Samali A. The eIF2alpha kinases: their structures and functions. Cell Mol Life Sci. 2013;70(19):3493-511; DOI: 10.1007/s00018-012-1252-6.

7. Crosby JS, Chefalo PJ, Yeh I, Ying S, London IM, Leboulch P, et al. Regulation of hemoglobin synthesis and proliferation of differentiating erythroid cells by heme-regulated eIF-2alpha kinase. Blood. 2000;96(9):3241-8;

8. McEwen E, Kedersha N, Song B, Scheuner D, Gilks N, Han A, et al. Heme-regulated inhibitor kinase-mediated phosphorylation of eukaryotic translation initiation factor 2 inhibits translation, induces stress granule formation, and mediates survival upon arsenite exposure. J Biol Chem. 2005;280(17):16925-33; DOI: 10.1074/jbc.M412882200.

9. Dong J, Qiu H, Garcia-Barrio M, Anderson J, Hinnebusch AG. Uncharged tRNA activates GCN2 by displacing the protein kinase moiety from a bipartite tRNA-binding domain. Mol Cell. 2000;6(2):269-79; DOI: 10.1016/s1097-2765(00)00028-9.

10. Emara MM, Fujimura K, Sciaranghella D, Ivanova V, Ivanov $P$, Anderson P. Hydrogen peroxide induces stress granule formation independent of eIF2alpha phosphorylation. Biochem Biophys Res Commun 2012;423(4):763-9; DOI: 10.1016/j.bbrc.2012.06.033.

11. Szaflarski W, Fay MM, Kedersha N, Zabel M, Anderson P, Ivanov P. Vinca alkaloid drugs promote stress-induced translational repression and stress granule formation. Oncotarget. 2016;7(21):30307-22; DOI: 10.18632/oncotarget.8728.

12. Arimoto-Matsuzaki K, Saito H, Takekawa M. TIA1 oxidation inhibits stress granule assembly and sensitizes cells to stress-induced apoptosis. Nat Commun. 2016;7:10252; DOI: 10.1038/ncomms10252.

13. Aulas A, Lyons SM, Fay MM, Anderson P, Ivanov P. Nitric oxide triggers the assembly of "type II" stress granules linked to decreased cell viability. Cell Death Dis. 2018;9(11):1129; DOI: 10.1038/ s41419-018-1173-x.

14. Ivanov P, Kedersha N, Anderson P. Stress Granules and Processing Bodies in Translational Control. Cold Spring Harb Perspect Biol. 2018; DOI: 10.1101/cshperspect.a032813.

15. Nunes C, Mestre I, Marcelo A, Koppenol R, Matos CA, Nobrega C. MSGP: the first database of the protein components of the mammalian stress granules. Database (Oxford). 2019;2019; DOI: 10.1093/database/ baz031.

16. Protter DS, Parker R. Principles and Properties of Stress Granules. Trends Cell Biol. 2016; DOI: 10.1016/j.tcb.2016.05.004.

17. Kedersha N, Panas MD, Achorn CA, Lyons S, Tisdale S, Hickman T, et al. G3BP-Caprin1-USP10 complexes mediate stress granule condensation and associate with 40S subunits. J Cell Biol. 2016;212(7):845-60; DOI: $10.1083 / j \mathrm{cb} .201508028$

18. Sanders DW, Kedersha N, Lee DSW, Strom AR, Drake V, Riback JA, et al. Competing Protein-RNA Interaction Networks Control Multiphase Intracellular Organization. Cell. 2020;181(2):306-24 e28; DOI: 10.1016/j. cell.2020.03.050.

19. Siang DTC, Lim YC, Kyaw AMM, Win KN, Chia SY, Degirmenci U, et al. The RNA-binding protein HuR is a negative regulator in adipogenesis. Nat Commun. 2020;11(1):213; DOI: 10.1038/s41467-019-14001-8.

20. Akins MR, Berk-Rauch HE, Kwan KY, Mitchell ME, Shepard KA, Korsak LI, et al. Axonal ribosomes and mRNAs associate with fragile $\mathrm{X}$ granules in adult rodent and human brains. Hum Mol Genet. 2017;26(1):192-209; DOI: $10.1093 / \mathrm{hmg} / \mathrm{ddw} 381$.

21. Kim TH, Tsang B, Vernon RM, Sonenberg N, Kay LE, Forman-Kay JD. Phospho-dependent phase separation of FMRP and CAPRIN1 recapitulates regulation of translation and deadenylation. Science. 2019;365(6455):825-9; DOI: 10.1126/science.aax4240.

22. Lai A, Valdez-Sinon AN, Bassell GJ. Regulation of RNA granules by FMRP and implications for neurological diseases. Traffic. 2020;21(7):454-62; DOI: $10.1111 /$ tra.12733.

23. Kedersha N, Ivanov P, Anderson P. Stress granules and cell signaling: more than just a passing phase? Trends Biochem Sci. 2013;38(10):494506; DOI: 10.1016/j.tibs.2013.07.004

24. Lloyd RE. Regulation of stress granules and P-bodies during RNA virus infection. Wiley Interdiscip Rev RNA. 2013;4(3):317-31; DOI: 10.1002/ wrna.1162.

25. Wehner KA, Schutz S, Sarnow P. OGFOD1, a novel modulator of eukaryotic translation initiation factor 2alpha phosphorylation and the cellular response to stress. Mol Cell Biol. 2010;30(8):2006-16; DOI: 10.1128/MCB.01350-09.

26. Arimoto K, Fukuda H, Imajoh-Ohmi S, Saito H, Takekawa M. Formation of stress granules inhibits apoptosis by suppressing stress-responsive MAPK pathways. Nat Cell Biol. 2008;10(11):1324-32; DOI: 10.1038/ ncb1791. 
27. Qiu Y, Mao T, Zhang Y, Shao M, You J, Ding Q, et al. A crucial role fo RACK1 in the regulation of glucose-stimulated IRE1alpha activation in pancreatic beta cells. Sci Signal. 2010;3(106):ra7; DOI: 10.1126/ scisignal.2000514.

28. Efeyan A, Zoncu R, Sabatini DM. Amino acids and mTORC1: from lysosomes to disease. Trends Mol Med. 2012;18(9):524-33; DOI: 10.1016/j. molmed.2012.05.007.

29. Sancak Y, Thoreen CC, Peterson TR, Lindquist RA, Kang SA, Spooner E, et al. PRAS40 is an insulin-regulated inhibitor of the mTORC1 protein kinase. Mol Cell. 2007;25(6):903-15; DOI: 10.1016/j.molcel.2007.03.003.

30. Takahara T, Maeda T. Transient sequestration of TORC1 into stress granules during heat stress. Mol Cell. 2012;47(2):242-52; DOI: 10.1016/j. molcel.2012.05.019.

31. Chi X, Wang S, Huang Y, Stamnes M, Chen JL. Roles of rho GTPases in intracellular transport and cellular transformation. Int J Mol Sci. 2013;14(4):7089-108; DOI: 10.3390/ijms14047089.

32. Ongusaha PP, Qi HH, Raj L, Kim YB, Aaronson SA, Davis RJ, et al. Identification of ROCK1 as an upstream activator of the JIP-3 to JNK signaling axis in response to UVB damage. Sci Signal. 2008;1(47):ra14; DOI: 10.1126/scisignal.1161938.

33. Sahoo PK, Murawala P, Sawale PT, Sahoo MR, Tripathi MM, Gaikwad SR, et al. Wnt signalling antagonizes stress granule assembly through a Dishevelled-dependent mechanism. Biol Open. 2012;1(2):109-19; DOI 10.1242/bio.2011023.

34. Mahboubi H, Stochaj U. Cytoplasmic Stress Granules: Dynamic Modulators of Cell Signaling and Disease. Biochim Biophys Acta. 2017; DOI: 10.1016/j.bbadis.2016.12.022.

35. Zhan Y, Wang H, Ning Y, Zheng H, Liu S, Yang Y, et al. Understanding the roles of stress granule during chemotherapy for patients with malignant tumors. Am J Cancer Res. 2020;10(8):2226-41;

36. Shaw RJ, Cantley LC. Ras, PI(3)K and mTOR signalling controls tumour cell growth. Nature. 2006;441(7092):424-30; DOI: 10.1038/ nature 04869 .

37. Bittencourt LFF, Negreiros-Lima GL, Sousa LP, Silva AG, Souza IBS, Ribeiro R, et al. G3BP1 knockdown sensitizes U87 glioblastoma cell line to Bortezomib by inhibiting stress granules assembly and potentializing apoptosis. J Neurooncol. 2019;144(3):463-73; DOI: 10.1007/ s11060-019-03252-6.

38. Somasekharan SP, El-Naggar A, Leprivier G, Cheng H, Hajee S, Grunewald $\mathrm{TG}$, et al. YB-1 regulates stress granule formation and tumor progression by translationally activating G3BP1. J Cell Biol. 2015;208(7):91329; DOI: $10.1083 /$ jcb.201411047.

39. Takahashi M, Higuchi M, Matsuki H, Yoshita M, Ohsawa T, Oie M, et al. Stress granules inhibit apoptosis by reducing reactive oxygen species production. Mol Cell Biol. 2013;33(4):815-29; DOI: 10.1128/ MCB.00763-12.

40. Adjibade P, Simoneau B, Ledoux N, Gauthier WN, Nkurunziza M, Khandjian EW, et al. Treatment of cancer cells with Lapatinib negatively regulates general translation and induces stress granules formation. PLoS One. 2020;15(5):e0231894; DOI: 10.1371/journal.pone.0231894.

41. Ackerman D, Simon MC. Hypoxia, lipids, and cancer: surviving the harsh tumor microenvironment. Trends Cell Biol. 2014;24(8):472-8; DOI 10.1016/j.tcb.2014.06.001

42. Sim E, Irollo E, Grabocka E. Evaluating Stress Granules in Pancreatic Cancer In Vitro and In Vivo. Methods Mol Biol. 2019;1882:183-95; DOI 10.1007/978-1-4939-8879-2_17.

43. Adjibade P, St-Sauveur VG, Quevillon Huberdeau M, Fournier MJ, Savard $\mathrm{A}$, Coudert $\mathrm{L}$, et al. Sorafenib, a multikinase inhibitor, induces formation of stress granules in hepatocarcinoma cells. Oncotarget 2015;6(41):43927-43; DOI: 10.18632/oncotarget.5980.

44. Vilas-Boas Fde A, da Silva AM, de Sousa LP, Lima KM, Vago JP, Bittencourt LF, et al. Impairment of stress granule assembly via inhibition of the eIF2alpha phosphorylation sensitizes glioma cells to chemotherapeutic agents. J Neurooncol. 2016;127(2):253-60; DOI: 10.1007/ s11060-015-2043-3.

45. Zhao J, Fu X, Chen H, Min L, Sun J, Yin J, et al. G3BP1 interacts with YWHAZ to regulate chemoresistance and predict adjuvant chemotherapy benefit in gastric cancer. Br J Cancer. 2021;124(2):425-36; DOI: 10.1038/s41416-020-01067-1.

46. Legrand N, Dixon DA, Sobolewski C. Stress granules in colorectal cancer: Current knowledge and potential therapeutic applications. World J Gastroenterol. 2020;26(35):5223-47; DOI: 10.3748/wjg.v26.i35.5223.

47. Wolozin B, Ivanov P. Stress granules and neurodegeneration. Nat Rev Neurosci. 2019;20(11):649-66; DOI: 10.1038/s41583-019-0222-5.

48. Bentmann E, Haass C, Dormann D. Stress granules in neurodegeneration--lessons learnt from TAR DNA binding protein of $43 \mathrm{kDa}$ and fused in sarcoma. FEBS J. 2013;280(18):4348-70; DOI: 10.1111/febs.12287.

49. Ayala YM, Pantano S, D'Ambrogio A, Buratti E, Brindisi A, Marchetti C, et al. Human, Drosophila, and C.elegans TDP43: nucleic acid binding properties and splicing regulatory function. J Mol Biol. 2005;348(3):57588; DOI: 10.1016/j.jmb.2005.02.038.

50. Buratti E, Baralle FE. The multiple roles of TDP-43 in pre-mRNA processing and gene expression regulation. RNA Biol. 2010;7(4):420-9; DOI: 10.4161/rna.7.4.12205.

51. Colombrita C, Zennaro E, Fallini C, Weber M, Sommacal A, Buratti E, et al. TDP-43 is recruited to stress granules in conditions of oxidative insult. J Neurochem. 2009;111(4):1051-61; DOI: 10.1111/j.1471-4159.2009.06383.x

52. McDonald KK, Aulas A, Destroismaisons L, Pickles S, Beleac E, Camu W, et al. TAR DNA-binding protein 43 (TDP-43) regulates stress granule dynamics via differential regulation of G3BP and TIA-1. Hum Mol Genet. 2011;20(7):1400-10; DOI: 10.1093/hmg/ddr021.

53. Aulas A, Stabile S, Vande Velde C. Endogenous TDP-43, but not FUS contributes to stress granule assembly via G3BP. Mol Neurodegener 2012;7:54; DOI: 10.1186/1750-1326-7-54.

54. Neumann M, Sampathu DM, Kwong LK, Truax AC, Micsenyi MC, Chou TT, et al. Ubiquitinated TDP-43 in frontotemporal lobar degeneration and amyotrophic lateral sclerosis. Science. 2006;314(5796):130-3; DOI: 10.1126/science.1134108.

55. Arai T, Hasegawa M, Akiyama H, Ikeda K, Nonaka T, Mori H, et al. TDP43 is a component of ubiquitin-positive tau-negative inclusions in frontotemporal lobar degeneration and amyotrophic lateral sclerosis. Biochem Biophys Res Commun. 2006;351(3):602-11; DOI: 10.1016/j. bbrc.2006.10.093.

56. Khalfallah Y, Kuta R, Grasmuck C, Prat A, Durham HD, Vande Velde C. TDP-43 regulation of stress granule dynamics in neurodegenerative disease-relevant cell types. Sci Rep. 2018;8(1):7551; DOI: 10.1038/ s41598-018-25767-0.

57. Bosco DA, Lemay N, Ko HK, Zhou H, Burke C, Kwiatkowski TJ, Jr., et al. Mutant FUS proteins that cause amyotrophic lateral sclerosis incorporate into stress granules. Hum Mol Genet. 2010;19(21):4160-75; DOI $10.1093 / \mathrm{hmg} / \mathrm{ddq} 335$.

58. Gal J, Zhang J, Kwinter DM, Zhai J, Jia H, Jia J, et al. Nuclear localization sequence of FUS and induction of stress granules by ALS mutants. Neurobiol Aging. 2011;32(12):2323 e27-40; DOI: 10.1016/j. neurobiolaging.2010.06.010.

59. Ito D, Seki M, Tsunoda Y, Uchiyama H, Suzuki N. Nuclear transport impairment of amyotrophic lateral sclerosis-linked mutations in FUS/TLS. Ann Neurol. 2011;69(1):152-62; DOI: 10.1002/ana.22246.

60. Dormann D, Rodde R, Edbauer D, Bentmann E, Fischer I, Hruscha A, et al. ALS-associated fused in sarcoma (FUS) mutations disrupt Transportin-mediated nuclear import. EMBO J. 2010;29(16):2841-57; DOI: 10.1038/emboj.2010.143.

61. Li W, Li Y, Kedersha N, Anderson P, Emara M, Swiderek KM, et al. Cell proteins TIA-1 and TIAR interact with the 3' stem-loop of the West Nile virus complementary minus-strand RNA and facilitate virus replication. J Virol. 2002;76(23):11989-2000; DOI: 10.1128/ jvi.76.23.11989-12000.2002

62. Valiente-Echeverria F, Melnychuk L, Mouland AJ. Viral modulation of stress granules. Virus Res. 2012;169(2):430-7; DOI: 10.1016/j. virusres.2012.06.004.

63. White JP, Cardenas AM, Marissen WE, Lloyd RE. Inhibition of cytoplasmic mRNA stress granule formation by a viral proteinase. Cell Host Microbe. 2007;2(5):295-305; DOI: 10.1016/j.chom.2007.08.006.

64. Ng CS, Jogi M, Yoo JS, Onomoto K, Koike S, Iwasaki T, et al. Encephalomyocarditis virus disrupts stress granules, the critical platform for triggering antiviral innate immune responses. J Virol. 2013;87(17):9511-22; DOI: $10.1128 /$ JVI.03248-12.

65. Isler JA, Skalet AH, Alwine JC. Human cytomegalovirus infection activates and regulates the unfolded protein response. J Virol. 2005;79(11):68909; DOI: 10.1128/JVI.79.11.6890-6899.2005.

66. Arruebo M, Vilaboa N, Saez-Gutierrez B, Lambea J, Tres A, Valladares M, et al. Assessment of the evolution of cancer treatment therapies. Cancers (Basel). 2011;3(3):3279-330; DOI: 10.3390/cancers3033279.

67. Panas MD, Ivanov P, Anderson P. Mechanistic insights into mammalian stress granule dynamics. J Cell Biol. 2016;215(3):313-23; DOI: 10.1083/ jcb.201609081.

68. Gao X, Jiang L, Gong Y, Chen X, Ying M, Zhu H, et al. Stress granule: A promising target for cancer treatment. Br J Pharmacol. 2019;176(23):4421 33; DOI: $10.1111 /$ bph.14790.

69. Anderson P, Kedersha N, Ivanov P. Stress granules, P-bodies and cancer. Biochim Biophys Acta. 2015;1849(7):861-70; DOI: 10.1016/j. bbagrm.2014.11.009.

70. Franchini DM, Lanvin O, Tosolini M, Patras de Campaigno E, Cammas A, Pericart S, et al. Microtubule-Driven Stress Granule Dynamics Regulate Inhibitory Immune Checkpoint Expression in T Cells. Cell Rep. 2019;26(1):94-107 e7; DOI: 10.1016/j.celrep.2018.12.014. 